\title{
Responsiveness of hypothalamo- pituitary-adrenal axis to leptin is impaired in diet-induced obese rats
}

\author{
Andrew C. Shin ${ }^{1}$, Sheba M. J. MohanKumar², Priya Balasubramanian ${ }^{3}$, Madhu P. Sirivelu $^{3}$, Katrina Linning ${ }^{3}$,
} Andrew Woolcock ${ }^{3}$, Michelle James ${ }^{3}$ and Puliyur S. MohanKumar ${ }^{2}$

\begin{abstract}
Background/objectives: Diet-induced obese (DIO) rats have altered stress (HPA) axis activity compared to dietresistant (DR) rats when chronically exposed to a high-fat (HF) diet. Since stress axis is tightly regulated by leptin, an adipocyte-secreted hormone that is important for controlling body weight, we hypothesized that leptin action is impaired in DIO rats leading to alterations in HPA axis activity.
\end{abstract}

Subjects/methods: We intraperitoneally injected selectively bred DIO and DR rats with either saline or recombinant rat leptin. HPA axis activity was assessed by measuring norepinephrine (NE) in the paraventricular nucleus (PVN), corticotropin-releasing hormone $(\mathrm{CRH})$ in the median eminence, and serum corticosterone (CORT). To test if HF exposure duration and the corresponding increase in leptin differentially affects HPA axis activity, we placed animals on a chow or HF diet for 1 or 6 weeks.

Results: Leptin injection significantly increased serum leptin levels in both DIO and DR animals. It also reduced PVN NE in both groups, indicating that noradrenergic neurons in both groups remain responsive to leptin. HF diet durationdependently increased serum leptin only in DIO animals whereas PVN NE increased in both groups. While DR rats responded to HF diet by increasing $\mathrm{CRH}$ and CORT at both time-points, responses in DIO rats varied, suggesting that they have altered HPA axis activity that may be dependent on HF-induced leptin levels and/or signaling. To understand the underlying mechanisms, we measured pSTAT-3, a marker of leptin signaling, in brainstem noradrenergic neurons and found reduced PSTAT-3 in A1 region of HF-fed DIO rats. We also found higher serum free fatty acids (FFAs) and a pro-inflammatory cytokine, IL-1 $\beta$.

Conclusions: Collectively, these findings reveal that DIO rats have inherent neuroendocrine impairment in NE-HPA axis circuitry that worsens with the extent of HF diet exposure, possibly due to brainstem leptin resistance and/or elevated circulating FFAs and IL-1 $\beta$.

Correspondence: Puliyur S. MohanKumar (psmohan@uga.edu)

'Department of Nutritional Sciences, College of Human Sciences, Texas Tech University, Texas, TX 79409, USA

${ }^{2}$ Department of Veterinary Biosciences and Diagnostic Imaging, University of Georgia, Athens, GA 30602, USA

Full list of author information is available at the end of the article.

\section{Introduction}

Diet-induced obesity (DIO) is a serious health condition that has been affecting individuals of all ages. Stress is one of many causes that has long been implicated as a contributing factor for the development of obesity ${ }^{1}$. Stress alters brain circuits to favor consumption of palatable, high-energy foods, promotes lipid accumulation in liver, and increases insulin secretion to promote

\section{(c) The Author(s) 2019}

(c) Open Access This article is licensed under a Creative Commons Attribution 4.0 International License, which permits use, sharing, adaptation, distribution and reproduction c. in any medium or format, as long as you give appropriate credit to the original author(s) and the source, provide a link to the Creative Commons license, and indicate if changes were made. The images or other third party material in this article are included in the article's Creative Commons license, unless indicated otherwise in a credit line to the material. If material is not included in the article's Creative Commons license and your intended use is not permitted by statutory regulation or exceeds the permitted use, you will need to obtain permission directly from the copyright holder. To view a copy of this license, visit http://creativecommons.org/licenses/by/4.0/. 
adiposity $^{2}$. Activation of the stress axis involves increased noradrenergic outflow to the paraventricular nucleus of the hypothalamus (PVN) that contains corticotropin-releasing hormone $(\mathrm{CRH})$ neurons. Stimulation of $\mathrm{CRH}$ neurons by norepinephrine (NE) results in $\mathrm{CRH}$ release in the median eminence (ME) that is transported to the anterior pituitary to stimulate corticotrophs to secrete adrenocorticotropic hormone $(\mathrm{ACTH})$. ACTH then acts on the adrenal cortex to stimulate secretion of corticosterone (CORT). Since stress plays a key role in driving anabolism, we had suspected that high-fat (HF) diet would further stimulate the stress axis, contributing to the perpetuation of a vicious cycle leading to diet-induced obesity. However, our previous work demonstrated that HF feeding for 6 weeks does not increase stress axis activity, but rather induces characteristic dysregulation of the stress axis in DIO rats $^{3}$. Specifically, HF feeding increased NE levels in the PVN of DIO rats, but failed to produce a corresponding increase in CRH and CORT as demonstrated in dietresistant (DR) rats.

HF diet increases fat mass and hence secretion of leptin from the adipose tissue ${ }^{4}$. Similarly, CORT increases leptin gene expression ${ }^{5}$ and stimulates leptin secretion ${ }^{6}$. Leptin, in turn, works as a negative feedback signal to suppress the stress axis. In support of this concept, studies have shown the ability of leptin to decrease NE release in the PVN (i.e., leptin action) and concurrently decrease serum corticosterone in rats ${ }^{7}, \mathrm{NE}$ efflux from the hypothalamus ${ }^{8}$, and to suppress $\mathrm{ACTH}$ and corticosterone in mice? Although HF feeding for 6 weeks does increase circulating leptin in DIO rats, the persistent increase in NE levels in the PVN suggests leptin insensitivity in NE neurons ${ }^{3}$. Evidence suggests that leptin resistance in neurons is most likely due to impaired downstream signaling that involves phosphorylated signal transducer and activator of transcription-3 (pSTAT-3) ${ }^{10}$ and suppressor of cytokine signaling-3 (SOCS-3) ${ }^{11}$, the negative feedback inhibitor of leptin signaling. Increases in circulating free fatty acids (FFAs), triglycerides, and/or pro-inflammatory cytokines may have independent effects in stimulating hypothalamic $\mathrm{NE}$ or inducing leptin insensitivity ${ }^{12-18}$. We hypothesized that leptin resistance in NE neurons (1) is mediated through downstream pSTAT-3 pathway, (2) is progressive with increased duration of HF feeding, and (3) is associated with changes in circulating FFAs and cytokines.

Therefore, in the present study, we treated DIO and DR rats with a single dose of recombinant rat leptin or subjected the rats to HF feeding for 1 or 6 weeks. We followed the changes in different arms of the stress axis and investigated the effects of HF diet exposure on serum FFAs and cytokine levels, as well as pSTAT-3 expression in brainstem noradrenergic neurons to determine the site of leptin insensitivity.

\section{Methods \\ Animals}

Breeding pairs of polygenically obese DIO (i.e., obesityprone) and DR rats were obtained from Charles River Laboratories, Inc. (Wilimington, MA). They were housed in temperature-controlled $\left(23 \pm 2{ }^{\circ} \mathrm{C}\right)$ rooms on a 12 :12-h light-dark schedule with ad libitum access to food and water. They were bred in our colony and offspring were weaned after 1 month. After weaning, the offspring were single-housed and fed standard chow diet (SC; Teklad 8640 diet; $3.11 \mathrm{kcal} / \mathrm{g}$, 5\% fat; Harlan, Indianapolis, IN) until they were used in the experiments. Sample size for each group is chosen based on prior studies in our laboratory with similar protocols. Animals were handled every day to minimize any potential stress before and during the experiments. All studies were conducted in accordance with the National Institutes of Health's Guide for the Care and Use of Laboratory Animals and the protocol was approved by the institutional animal care and use committee at Michigan State University.

\section{Treatment}

In first experiment, overnight-fasted 3-month old male DIO and DR rats (6-8 per group) were either injected intraperitoneally with $250 \mu \mathrm{l}$ of saline or $500 \mu \mathrm{g}$ of rat recombinant leptin (R\&D systems, Minneapolis, MN) dissolved in $250 \mu \mathrm{l}$ of saline. Rats were sacrificed $5 \mathrm{~h}$ later and serum was separated from trunk blood for leptin and CORT measurements. We followed this protocol because our previous time-course study ${ }^{7}$ has clearly demonstrated that the HPA axis, as assessed by serum corticosterone, in normal Sprague-Dawley rats is effectively suppressed starting at $3 \mathrm{~h}$, but is maximally decreased $5 \mathrm{~h}$ after an ip injection of leptin. PVN NE levels were also found to be decreased from as early as $30 \mathrm{~min}$ to $5 \mathrm{~h}$ post-leptin treatment $^{7,19}$. Brains were removed and frozen immediately on dry ice and stored at $-70^{\circ} \mathrm{C}$ for processing as described below. In second experiment, 3-month old male DIO and DR rats (6-8 per group) were fed either SC diet or HF diet for either 1 or 6 weeks. The HF diet contained 20\% protein, $35 \%$ carbohydrate, and $45 \%$ calories as fat with an energy density of $4.73 \mathrm{kcal} / \mathrm{g}$ (D12451; Research Diets Inc., New Brunswick, NJ). Food intake and body weight were measured on a weekly basis until the end of treatment. At the end of the treatment, the rats were sacrificed after $2-3 \mathrm{~h}$ fasting ( 10am), and their brains were collected, frozen in dry ice, and stored at $-70^{\circ} \mathrm{C}$. Trunk blood was collected, the serum was separated, and stored at $-20^{\circ} \mathrm{C}$ until RIA and ELISA analyses. Visceral fat (retroperitoneal, epidydimal, perirenal) was removed from the carcass and weighed.

\section{Palkovits' microdissection}

The PVN and ME, as well as noradrenergic nuclei from the brainstem ( $\mathrm{A} 1$, ventrolateral medulla; $\mathrm{A} 2$, nucleus 
tractus solitarus; and A6, locus coeruleus) were isolated from $300 \mu \mathrm{m}$ brain sections via micropunch tools, as described before ${ }^{3,19}$ using a rat brain atlas ${ }^{20}$. Care was taken to include all subdivisions of the nuclei from multiple serial sections. The ME was stored as such at $-70^{\circ} \mathrm{C}$ until analysis for $\mathrm{CRH}$ concentrations using ELISA. The PVN was stored in $0.1 \mathrm{M} \mathrm{HClO}_{4}$ at $-70{ }^{\circ} \mathrm{C}$ and analyzed for NE concentrations using HPLC-EC. Brainstem tissue punches were stored in $-70^{\circ} \mathrm{C}$ for western blot analysis.

\section{HPLC-EC}

The HPLC-EC system and the details of the mobile phase have been described previously ${ }^{8,19}$. Briefly, the system consisted of a Shimadzu LC-10 AT/VP pump, a phase II, $5 \mu \mathrm{m}$ ODS reverse-phase, C-18 column (Phenomenex, Torrance, CA), a glassy carbon electrode (Bioanalytical Systems, West Lafayette, IN) placed inside a Shimadzu CTO-10 AT/VP column oven at $37^{\circ} \mathrm{C}$, and a LC-4C amperometric detector (Bioanalytical Systems, West Lafayette, IN) connected to a computer with the class VP chromatopac software (Shimadzu, Columbia, MD). The mobile phase was pumped at a flow rate of $1.8 \mathrm{ml} / \mathrm{min}$. The range of the detector was $1.0 \mathrm{nA}$ full scale, and the potential of the working electrode was $0.65 \mathrm{~V}$. At the time of HPLC analysis, microdissected tissue samples were thawed and homogenized in $150 \mu \mathrm{l}$ of $0.1 \mathrm{M} \mathrm{HClO}_{4}$ using a micro-ultrasonic cell disruptor (Kontes, Vineland, NJ) and centrifuged at $10,000 \times g$ for $10 \mathrm{~min} .120 \mu \mathrm{l}$ of the supernatant was mixed with $30 \mu \mathrm{l}$ of the internal standard $(0.05 \mathrm{M}$ dihydroxybenzylamine) and $125 \mu \mathrm{l}$ of this mixture was injected into the HPLC system. The sensitivity of the system was $<1 \mathrm{pg}$. NE concentrations were expressed as $\mathrm{pg} / \mu \mathrm{g}$ protein.

\section{Western blots}

Noradrenergic nuclei from the brainstem were homogenized in lysis solution (Sigma Aldrich, St. Louis, MO) and protein concentrations were determined using a micro BCA assay (Pierce, Rockford, IL). Equal quantities of protein $(20 \mu \mathrm{g})$ were loaded on to SDS-PAGE gels (NuPAGE, Invitrogen, Carlsbad, CA) and separated. Gels were blotted onto nitrocellulose membranes and the membranes were probed with pSTAT-3 antibody (1:1000; goat-polyclonal; Santa Cruz Biotechnology, Dallas, TX) and GAPDH antibody (1:2000; mouse-monoclonal; Sigma-Aldrich, St. Louis, $\mathrm{MO})$. After washing, the membranes were incubated in blocking solution containing goat anti-rabbit DyLight 800 and goat anti-mouse DyLight 680 secondary antibodies (1:5000; Thermo Fisher Scientific; Waltham, MA). Bands were visualized using an Odyssey imaging system (Li-COR biosciences, Lincoln, NE).

\section{ELISA for leptin, CRH, and IL-1 $\beta$}

Serum leptin was measured in duplicate using a commercial ELISA kit (TiterZyme Kits, Assay Design, Ann
Arbor, MI) according to the manufacturer's specifications. The plates were read at $450 \mathrm{~nm}$ using an ELISA reader (ELx800, BioTek Instruments, Inc., Winooski, VT). The sensitivity of the kit was $46.7 \mathrm{pg} / \mathrm{ml}$. CRH EIA kit (Phoenix Pharmaceuticals, Inc., Belmont, CA) was used to determine $\mathrm{CRH}$ protein in the ME. The assay had a minimum sensitivity of $0.30 \mathrm{ng} / \mathrm{ml}$. Protein concentrations in the tissue were measured and $\mathrm{CRH}$ was expressed as $\mathrm{ng} / \mu \mathrm{g}$ protein. Inter-assay variability was $<5 \%$. IL- $1 \beta$ was measured using a commercially available kit (Millipore, MA).

\section{Radioimmunoassay for CORT}

A double-antibody radioimmunoassay for CORT was performed using a tracer and standards from EMD Millipore (Billerica, MA) and indigenous primary and secondary antibodies. The samples $(50 \mu \mathrm{l})$ were assayed in duplicate as described previously ${ }^{3,7}$. The sensitivity of the assay was $0.2 \mathrm{ng} / \mathrm{ml}$. The inter-assay variability was $<4 \%$.

\section{Circulating FFA profiles}

Fatty acids in the serum were separated and quantified as described before ${ }^{21}$. Total lipids from serum were extracted using hexane:ethanol (1:1) solvent mix by mixing equal volumes of the serum and the solvent mix, vortexing for $10 \mathrm{~min}$ and centrifuging at $2,095 \mathrm{~g}$ for $10 \mathrm{~min}$ at room temperature. Fatty acids were transesterified and methylated using $20 \%$ methanolic hydrochloric acid for $2 \mathrm{~h}$ at $90^{\circ} \mathrm{C}$. Methylated extracts were analyzed using a Clarus 500 gas chromatography apparatus (Perkin Elmer, Waltham, MA) and separated on a Supelco SP-2560 column $(100 \mathrm{~m} \times 25 \mathrm{~mm}$ with a film thickness of $0.2 \mu \mathrm{m}$; Supelco, Bellefonte, PA). Purified methyl esters were used as standards (Nu-Chek Prep, Elysian, MN). Concentrations were expressed as $\mathrm{mg} / \mathrm{dl}$.

\section{Statistical analysis}

In leptin experiment, changes in $\mathrm{NE}, \mathrm{CRH}$, and serum hormones were analyzed by 2-way ANOVA whereas serum lipid levels in addition to these analytes in HF experiment were analyzed by 3-way ANOVA. Serum cytokine and protein expression in the brainstem were analyzed by 2 -way ANOVA. Weekly caloric intake differences in DIO \& DR animals after SC or HF diet exposure were analyzed by repeated measures 2-way ANOVA. Average calorie intake and feed efficiency were analyzed by 3-way ANOVA. All ANOVAs were followed by post-hoc Fisher's LSD test. Body weight (BW) at the end of treatment, BW gain/week during the treatment period, total white adipose tissue (visceral fat) weight, and the fat/BW ratio between DIO and DR animals within each dietary treatment were analyzed by student's t-test. 


\section{Results}

Exogenous leptin induces differential stress axis activity in DIO and DR rats

A single ip injection of recombinant rat leptin after overnight fasting increased serum leptin levels $(\mathrm{ng} / \mathrm{ml}$; Mean \pm SE) by $\sim 4$ fold in DIO rats $(8.26 \pm 1.9)$ and produced a 2-fold increase in DR rats $(4.23 \pm 0.5)$ compared to saline-treated controls $(1.8 \pm 0.3$ and $1.2 \pm$ 0.1 in DIO and DR respectively; Fig. 1a). This produced a corresponding decrease in PVN NE concentrations $(\mathrm{pg} / \mu \mathrm{g}$ protein; Mean $\pm \mathrm{SE})$ in both DIO $(13.4 \pm 0.5)$ and $\mathrm{DR}$ rats $(10.4 \pm 0.3)$ compared to controls $(24.9 \pm 2$ and $25.3 \pm 2.2$ in DIO and DR rats respectively; Fig. 1b). Interestingly, while CRH levels in the $\mathrm{ME}(\mathrm{ng} / \mu \mathrm{g}$ protein; Mean \pm SE) were reduced in leptin-treated DR rats $(11.8 \pm 2.1)$ compared to controls $(23.5 \pm 2.7 ; p<0.05)$, no change was noted in DIO rats with leptin treatment $(21.7 \pm 8.2$ vs. $17.2 \pm 1.5$ in controls; Fig. 1c). Serum CORT levels ( $\mathrm{ng} / \mathrm{ml}$; Mean $\pm \mathrm{SE}$ ) also showed a trend of decrease after leptin treatment in DR rats only (Fig. 1d). Collectively, these findings demonstrate that unlike DR rats that display intact leptin action on noradrenergic neurons and NE-HPA axis circuitry, DIO rats have uncoupling between NE and HPA axis activity, suggesting inherent neuroendocrine changes in DIO rats that are independent of chronic HF diet exposure as shown in our earlier study ${ }^{3}$.
Food intake and feed efficiency with 1 or 6 weeks of SC or HF diet

In the 1 week groups, cumulative chow intake (kcal) was higher in DIO compared to DR rats starting on the third day and remained higher at the end of the week (Fig. 2a). Cumulative HF intake was greater in DIO rats as early as the second day that continued throughout. A similar trend was observed for rats exposed to 6 weeks of chow or HF diet (Fig. 2b). Cumulative food intake was higher in both chow and HF-fed DIO rats compared to their DR counterparts. This is likely attributed to an increased daily average food intake in DIO vs. DR rats, and between HF vs. SC feeding (Fig. 2c). In agreement with earlier findings by Levin and colleagues ${ }^{22}$, there were no significant differences in feed efficiency between DIO and DR rats across treatments (Fig. 2d).

\section{Body weight and adiposity in DIO and DR rats after 1 or 6 weeks of SC or HF diet}

BW gain is expressed per week to eliminate confusion between different duration of treatments. Even with chow diet, DIO rats gained significantly more body weight per week (mean \pm SE; g; $21.1 \pm 0.6$ ) than DR rats $(15.8 \pm 0.7$; $p<0.001)$. One week of HF exposure did not produce any significant change in the BW gain between the two phenotypes $(p<0.08)$. However, prolonging the duration of HF diet to 6 weeks significantly increased BW gain in DIO
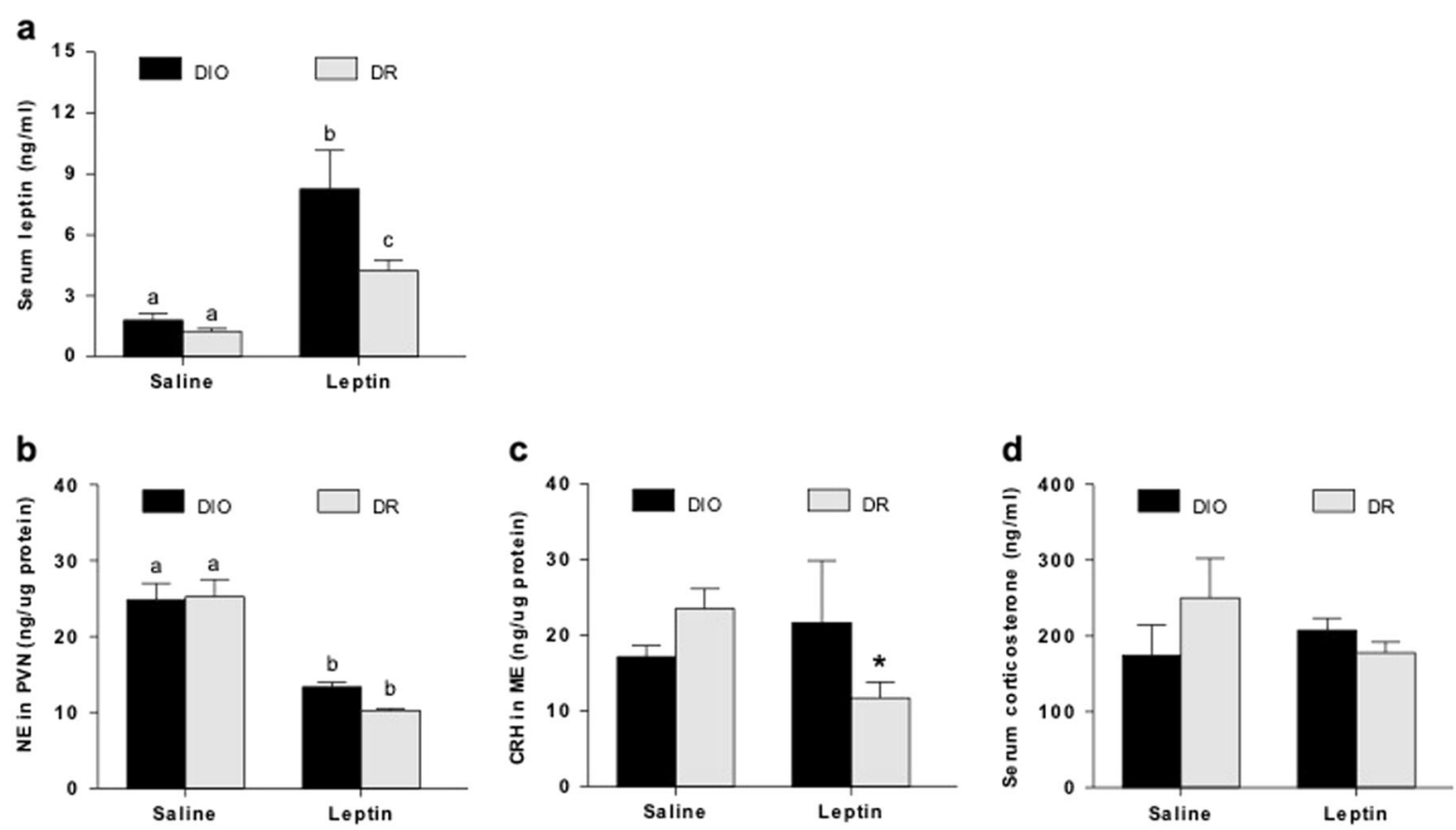

Fig. 1 Effects of a single leptin injection on serum leptin levels and HPA axis activity in DIO and DR rats. $\mathbf{a}$, $\mathbf{b}$ Note the marked reduction in PVN NE levels in both DIO and DR rats, indicating normal responsiveness to exogenous leptin. $\mathbf{c}$, $\mathbf{d}$ Decrease in ME CRH or serum corticosterone in DR rats following leptin injection is not observed in DIO rats. Bars with different notations are significantly different from each other ( $p<0.05)$. ${ }^{*}$ indicates significant difference from the rest of the groups $(p<0.05)$. 6-8 rats/group 

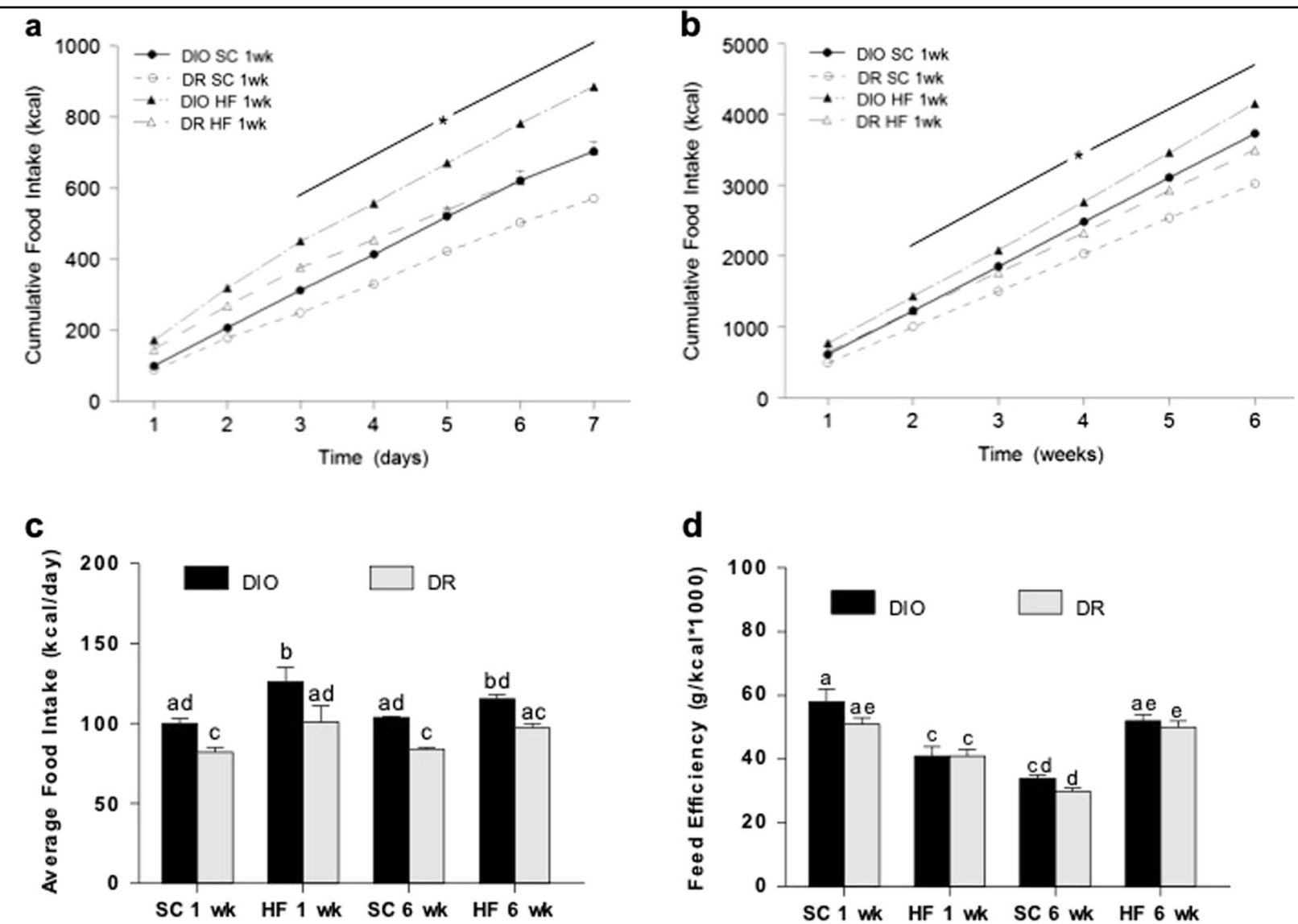

Fig. 2 Food intake in DIO and DR rats. The effect of standard chow (SC) or high fat (HF) diet for (a) 1 week or (b) 6 weeks on cumulative food intake. Food intake in HF-fed animals were significantly different from chow-fed animals. c Average food intake/day in the different treatment groups. d Average feed efficiency. ${ }^{*}$ indicates significant difference between DIO and DR group on the same diet. Bars with different notations are significantly different from each other $(p<0.05) .6-8$ rats/group

(35.9 \pm 1.3$)$ compared to DR animals $(29.7 \pm 1.2 ; p<0.05$; see Supplementary information Table S1).

\section{NE-HPA axis responsiveness to leptin is impaired after acute HF feeding and is further exacerbated after prolonged HF feeding in DIO rats}

While serum leptin levels ( $\mathrm{ng} / \mathrm{ml}$; Mean $\pm \mathrm{SE}$ ) did not change in DIO rats under SC diet $(2.4 \pm .3$ vs. $2.6 \pm 0.2$ at 1 and 6 weeks; Fig. 3a), HF feeding for 1 week significantly increased $(3.9 \pm 0.2)$ and 6 weeks further elevated serum leptin $(6.4 \pm 0.3 ; p<0.05)$. However, leptin levels were not altered in DR rats and this was independent of treatment duration. Next, we assessed HPA axis circuitry in these animals. In spite of no change in PVN NE between DIO and DR rats across treatments (Fig. 3b), 6 weeks of HF diet increased NE levels in both DIO and DR rats. HF feeding for 1 week, but not 6 weeks, markedly increased $\mathrm{ME} \mathrm{CRH} \mathrm{(ng/ \mu g} \mathrm{protein;} \mathrm{Mean} \pm$ SE; $45.8 \pm 11.51 \mathrm{wk}$ vs. $18.5 \pm 3.06 \mathrm{wks})$ compared to that under SC diet (18.6 \pm $7.1 ; p<0.05$ ) in DIO rats. (Fig. 3c). On the contrary, $\mathrm{CRH}$ levels in DR rats increased after 1 week of HF diet
$(31.9 \pm 6.3)$ and continued to increase after 6 weeks $(45.1 \pm 5.2)$ compared to those under SC diet (19.5 \pm 7.5 ; $p<0.05)$. Serum CORT levels ( $\mathrm{ng} / \mathrm{ml}$; Mean $\pm \mathrm{SE}$ ) elevated similarly in both DIO and DR rats after 1 week of HF diet compared to those in SC-fed animals (Fig. 3d). However, after 6 weeks of HF feeding, CORT continued to increase only in DR rats $(488.7 \pm 36.6$ vs. $384.5 \pm 12.6$ $1 \mathrm{wk})$ and not in DIO rats. It was in fact significantly lowered in DIO rats $(270.5 \pm 49.26 \mathrm{wks}$ vs. $409.7 \pm 41.3$ $1 w k ; p<0.05)$. These findings suggest that acute HF feeding impairs leptin action to suppress noradrenergic activity in DIO rats, and prolonged HF feeding further worsens the coupling of NE-HPA axis circuitry. Results from NE, CRH and CORT in response to exogenous leptin or HF treatment in DIO and DR rats are summarized in Supplementary information Table S2.

PSTAT-3 expression is reduced in brainstem noradrenergic neurons in DIO rats

To determine possible reasons for the inability of endogenous leptin to suppress PVN NE, we measured 

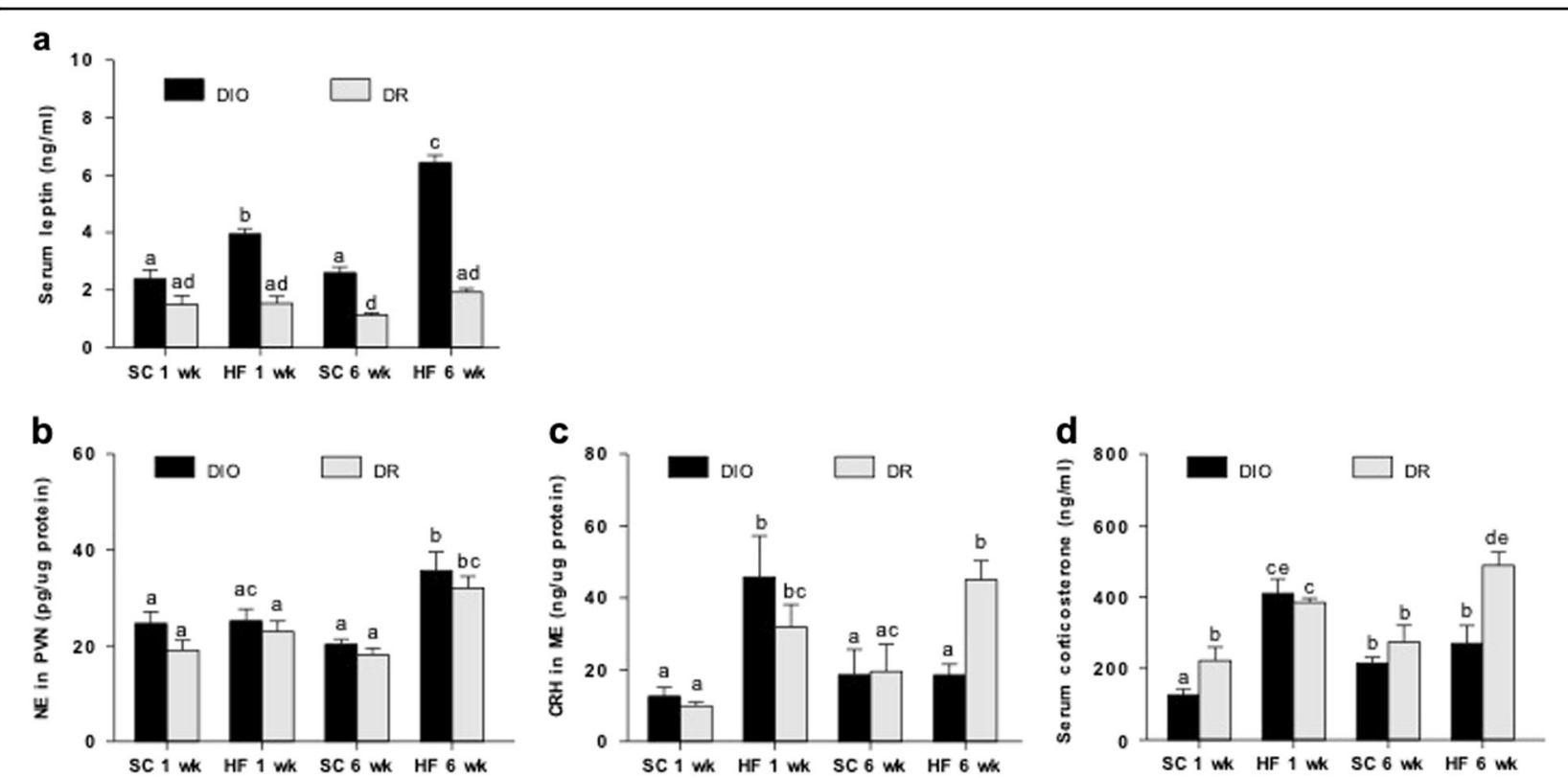

Fig. 3 Effects of different duration of HF diet exposure on serum leptin levels and HPA axis activity in DIO and DR rats. a, $b$ Note that PVN NE levels in DIO rats are elevated after 6 weeks of HF in spite of higher leptin levels. c, $\mathbf{d}$ DR rats have an activated stress axis in response to HF diet exposure, while DIO rats do not. Bars with different notations are significantly different from each other $(p<0.05)$. $6-8$ rats/group

pSTAT-3 protein levels in specific brainstem areas to assess leptin signaling. These areas contain mostly noradrenergic neurons. The expression of pSTAT-3 (Fold change; Mean $\pm \mathrm{SE}$ ) was markedly reduced in $\mathrm{A} 1$ region of DIO rats $(0.29 \pm 0.06)$ compared to DR rats $(1.08 \pm 0.1$; $p<0.05)$ after 6 weeks of HF diet (Fig. 4a). This nucleus provides the majority of noradrenergic innervation to the PVN where CRH neurons are localized ${ }^{23}$. In contrast, a significant reduction in pSTAT-3 expression was observed in A2 region of DIO rats irrespective of diet (Fig. 4b). There was no change in A6 region (Fig. 4c).

\section{Circulating FFAs and IL-1 $\beta$ are increased in DIO rats after HF feeding}

Serum oleic acid levels $(\mathrm{mg} / \mathrm{dl}$; Mean $\pm \mathrm{SE})$ were significantly increased in DIO rats after HF diet, but only after 1 week of HF diet for DR rats (Fig. 5a). Linoleic acid levels were markedly increased in DIO rats compared to DR rats irrespective of diet and duration (Fig. 5b). Arachidonic acid levels similarly increased in DIO and DR rats under HF diet (Fig. 5c). Serum IL-1 $\beta$ (pg/ml; Mean \pm SE; Fig. 5d) increased significantly only in DIO rats under HF diet.

\section{Discussion}

While the metabolic phenotype of polygenically bred DIO and DR rats has been extensively studied previously by Levin and colleagues ${ }^{22,24,25}$, our knowledge on potential neuroendocrine changes in DIO rats is somewhat limited to alternations in amygdalar CRH/GR gene expression and circulating glucocorticoid levels ${ }^{26-28}$. The underlying mechanism of altered HPA axis activity is also not well understood. We have previously identified leptin as an important regulator of HPA axis through suppressing $\alpha$-adrenergic signaling ${ }^{7}$, and our comprehensive analysis of leptin and NE-HPA axis circuitry - NE in the $\mathrm{PVN}, \mathrm{CRH}$ in the ME, serum corticosterone - demonstrated an uncoupling within the system in DIO but not DR rats chronically exposed to HF $\operatorname{diet}^{3}$. It was not clear, however, if this phenomenon is due to inherent genetic differences between them or is a result of HF feedingrelated changes. We tested this by giving a single dose of systemic leptin injection in DIO and DR rats under chow diet, and placed them on a HF diet for either 1 or 6 weeks to observe HF-related neuroendocrine changes as a function of time. Our current study extends previous research and shows novel findings that (1) increased noradrenergic tone in DIO rats may be due to leptin resistance in the brainstem and (2) dysregulation of the HPA axis and impaired leptin action on PVN NE under HF diet worsens in a time-dependent manner.

A single leptin injection significantly increased circulating leptin levels in both DIO and DR rats, albeit higher levels found in DIO rats. This is in agreement with studies showing reduced clearance rate for leptin in obese vs. lean subjects and increased clearance following an acute fasting $^{29,30}$. This resulted in a marked decrease in PVN NE in both groups, a response very similar to what was observed in lean rats from previous studies ${ }^{7,19,31,32}$. The result also indicates that the brainstem noradrenergic system in DIO 

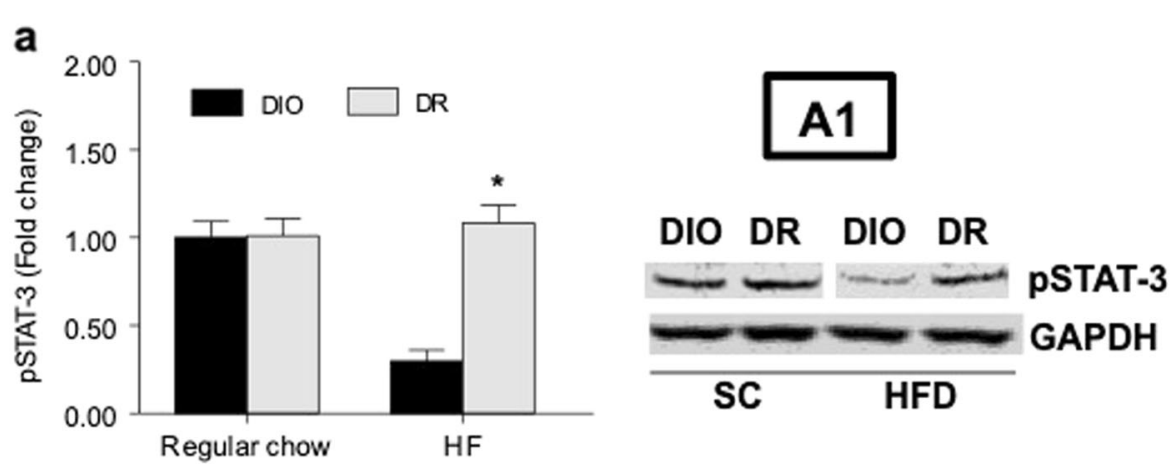

b
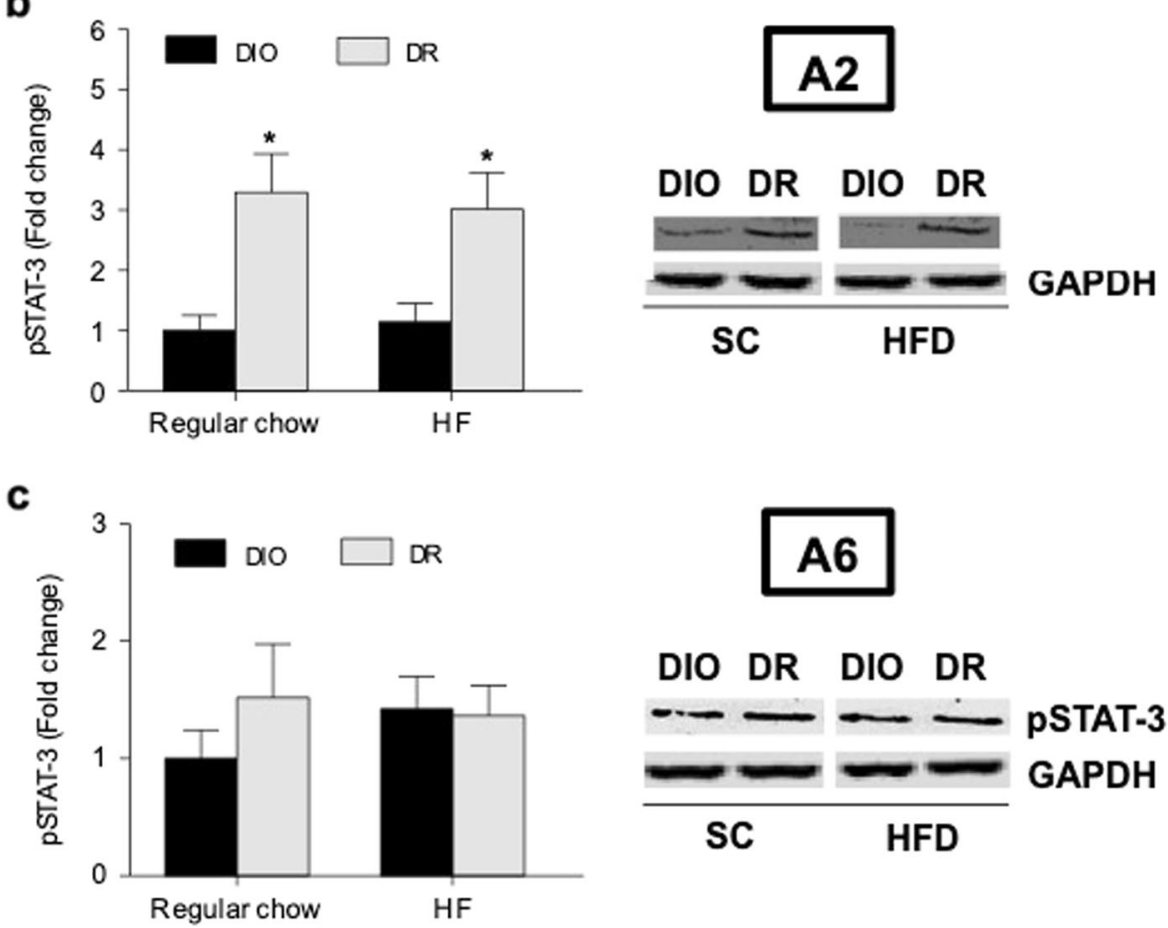

Fig. 4 Phosphorylation of STAT-3 in brainstem NE neurons after chow or HF diet exposure for 6 weeks. a Results from A1 noradrenergic nucleus; $\mathbf{b}$ A2 noradrenergic nucleus and $\mathbf{c}$ A6 noradrenergic nucleus. Data are normalized to GAPDH and expressed as a fold change from regular chow-fed DIO group. * indicates significant difference from the corresponding DIO group $(p<0.05)$. 6-8 rats/group

and DR rats remain sensitive to elevation of circulating leptin. Unlike NE, ME CRH levels and serum CORT decreased only in DR and remained unchanged in DIO rats (Supplementary Table S2 online), suggesting possible leptin/NE insensitivity in CRH neurons of DIO rats. While CRH is also expressed in other brain regions such as bed nucleus stria terminalis and amygdala ${ }^{33}$, the only source of $\mathrm{CRH}$ released into the ME before reaching the anterior pituitary is CRH neurons in the PVN. The lack of CORT response to leptin in DIO rats is unclear, but it is likely that other circulating factors such as proinflammatory cytokines and FFAs as discussed below counter the suppressive effects of leptin on CORT. The HPA responses in DR rats are very similar to what we have shown previously with leptin treatment in lean Sprague-Dawley rats ${ }^{7,19}$, suggesting that they have normal stress axis function.

Regardless of diets, DIO rats consumed more calories compared to DR rats, and their increase in fat mass likely accounts for the higher serum leptin seen especially under HF diet. On the other hand, we found a modest increase in fat mass in DR animals after 6 weeks of HF diet without affecting leptin levels. These results are in agreement with a previous finding showing that HF exposure increased serum leptin in DIO rats but not in DR rats ${ }^{22}$. When we examined the effects of HF diet on PVN NE, we found that NE levels were significantly higher in both genotypes after 6 weeks (Supplementary Table S2 online). 

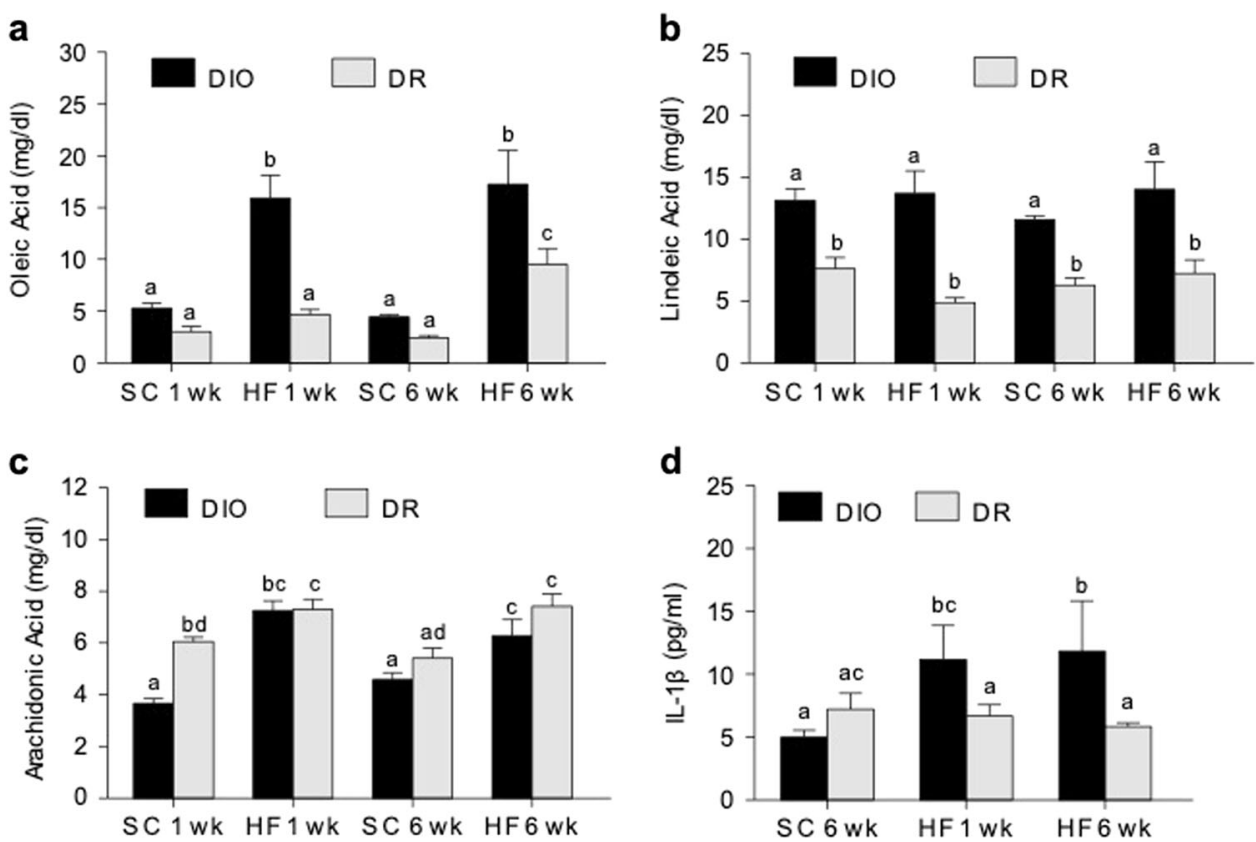

Fig. $\mathbf{5}$ Serum free fatty acids and IL-1 $\boldsymbol{\beta}$ levels. a Oleic acid, $\mathbf{b}$ Linoleic acid, and $\mathbf{c}$ Arachidonic acid levels were measured by GC-MS. $\mathbf{d}$ IL-1 $\beta$ levels were measured in SC-fed ( 6 weeks) rats compared to HF-fed DIO and DR rats. SC 1 wk group was not included because IL-1 $\beta$ was below detection level. Bars with different notations are significantly different from each other $(p<0.05)$. Note that DIO rats have higher levels of serum IL-1 $\beta$ after 1 or 6 weeks of HF diet exposure. 6-8 rats/group

Importantly, the inability of DIO rats to suppress PVN NE in spite of elevated leptin levels indicates impaired leptin action. Conversely, NE levels may increase in response to other HF-related circulating factors as discussed below. ME CRH and serum CORT increased in both groups after 1 week of HF diet, but only in DR rats after 6 weeks of HF diet. Collectively, the increase of PVN NE, CRH ME, and serum CORT in DR rats suggests that they perceive HF diet as a stressor. On the other hand, the continuous rise of PVN NE in the face of hyperleptinemia, and the initial, parallel rise in CRH and CORT and fall later in HF-fed DIO rats indicates dysregulated stress axis that becomes exacerbated as a function of time. Whether the reduced HPA axis activity seen in chronic HF-fed DIO rats leads to lower EE and differential substrate utilization that may be responsible for development of obesity needs further investigation.

Since functional leptin receptor $(\mathrm{ObRb})$ is present in all $\mathrm{A} 1, \mathrm{~A} 2$, and $\mathrm{A} 6$ regions in the brainstem ${ }^{34-37}$, we examined the possibility that leptin insensitivity localized to noradrenergic neurons in these areas contributes to higher NE levels in the PVN of DIO rats. Indeed, our results indicate that phosphorylation of STAT-3, a critical transcription factor for leptin signaling, decreases significantly in A1 region after HF feeding, and it is perhaps constitutively expressed at a low level in A2 region in DIO rats. Although we did not inject exogenous leptin to determine leptin signaling, we were able to significantly raise endogenous leptin in DIO rats by feeding a HF diet.
Diet-induced obesity is associated with a low-grade inflammation with increased circulating proinflammatory cytokines ${ }^{38,39}$. As STAT-3 can be phosphorylated not only by leptin but also by these cytokines including TNF- $\alpha$, IL- 6 , and IL- $1 \beta^{40}$, pSTAT- 3 may be expected to increase by elevated IL-1 $\beta$ we observed in HFfed DIO rats. However, our finding that it rather decreased strongly suggests that lower pSTAT-3 in the brainstem of DIO rats primarily reflects leptin resistance and not cytokine signaling. Whether or not acute leptinstimulated PSTAT-3 is lower after HF feeding in DIO rats would need to be tested.

Reduced leptin signaling in the brainstem of DIO rats may also be due to lack of leptin availability in the brain. Studies show that leptin's ability to cross the blood brain barrier can be impaired by HF feeding or circulating FFAs or triglycerides ${ }^{12,14,41}$. In this study, we found HF- and time-dependent increase in oleic acid and arachidonic acid in DIO rats. It is notable that arachidonic acid is also able to induce leptin resistance in the brain ${ }^{42}$. Serum linoleic acid was markedly elevated in DIO rats regardless of treatment, therefore it is difficult to assign a specific role of this FFA. Furthermore, most mono or polyunsaturated FAs such as above are also known to directly activate HPA axis ${ }^{13,17,18}$. Thus, these FFAs may participate in stimulating noradrenergic tone and/or HPA activity as seen in DIO rats via multiple mechanisms. Besides circulating FFAs, pro-inflammatory cytokines could also contribute to HPA axis dysfunction. In the 


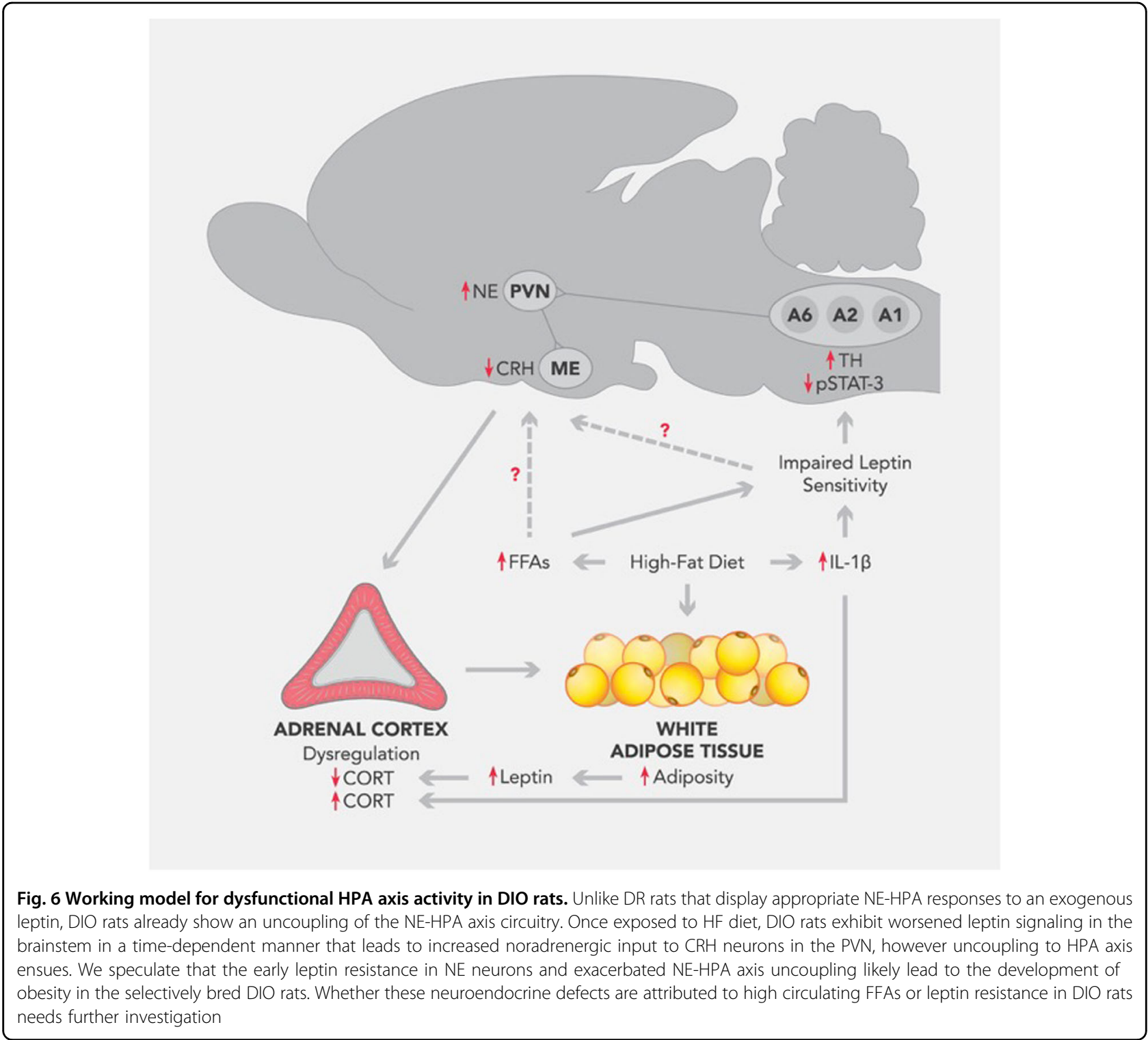

present study, serum IL-1 $\beta$ levels were markedly increased in DIO animals under HF diet that could have stimulatory effects on the HPA axis ${ }^{15,16}$. We speculate that HF-induced increase in IL-1 $\beta$ at least partly overrides leptin's suppressive effects on the HPA axis leading to its dysfunction in these rats.

Beyond its role in the control of HPA axis, leptin is better known for its action in the long-term regulation of body weight and food intake ${ }^{43,44}$. Intracerebroventricular (icv) $\mathrm{CRH}$ infusion lowers food intake in rats ${ }^{45,46}$ whereas $\mathrm{CRH}$ receptor antagonist attenuates leptin-induced reduction of food intake ${ }^{47}$, suggesting that $\mathrm{CRH}$ is critical for leptin-mediated appetite suppression. Interestingly, DIO rats develop leptin resistance in the ARC before exposed to $\mathrm{HF} \operatorname{diet}^{41}$, leading us to predict that $\mathrm{CRH}$ release from the PVN in HF-fed DIO rats will be lower compared to that in chow-fed DIO rats. On the contrary, our results demonstrate significantly increased ME CRH in 1 week HF-fed DIO rats compared their chow-fed counterparts, suggesting that this is most likely due to leptin resistance in the brainstem and not in the ARC. The potential contribution of intact leptin signaling in the ARC of DR rats on the overall ME CRH increase after HF feeding warrants further investigation.

In summary, the results from this study indicate that the noradrenergic system in the brainstem remains responsive to exogenous leptin in DIO and DR animals. However, when they are placed on a HF diet, there is a timedependent impairment of leptin action to suppress noradrenergic tone in the PVN of DIO rats that is most likely due to reduced pSTAT-3 expression in specific noradrenergic nuclei. DIO rats also appear to develop HPA 
axis dysfunction with prolonged HF diet exposure. Possible mediators of these phenomena include higher serum FFAs and pro-inflammatory cytokines such as IL-1 $\beta$. These may in turn override the NE-HPA suppressive effects of leptin promoting development of obesity in this polygenically obese model (Fig. 6).

\section{Acknowledgements}

This study was supported in part by NIH grant R01 AG027697 to PSM and NSF grant IBN0236385 to SMJM and PSM. ACS was supported by Biomedical Health Research Initiative grant, MSU. We thank Dr. Thomas Herdt, DCPAH, MSU for facilitating free fatty acid analysis.

\section{Author details}

${ }^{1}$ Department of Nutritional Sciences, College of Human Sciences, Texas Tech University, Texas, TX 79409, USA. ${ }^{2}$ Department of Veterinary Biosciences and Diagnostic Imaging, University of Georgia, Athens, GA 30602, USA.

${ }^{3}$ Neuroscience Program, Michigan State University, East Lansing, MI 48824, USA

\section{Conflict of interest}

The authors declare that they have no conflict of interest.

\section{Publisher's note}

Springer Nature remains neutral with regard to jurisdictional claims in published maps and institutional affiliations.

Supplementary Information accompanies this paper at (https://doi.org/ 10.1038/s41387-019-0076-y).

Received: 2 May 2018 Revised: 25 October 2018 Accepted: 6 November 2018

Published online: 18 March 2019

\section{References}

1. Sominsky, L. \& Spencer, S. J. Eating behavior and stress: a pathway to obesity. Front Psychol. 5, 434 (2014).

2. Sinha, R. \& Jastreboff, A. M. Stress as a common risk factor for obesity and addiction. Biol. Psychiatry 73, 827-835 (2013).

3. Shin, A. C. et al. Chronic exposure to a high-fat diet affects stress axis function differentially in diet-induced obese and diet-resistant rats. Int J. Obes. 34, 1218-1226 (2010)

4. Frederich, R. C. et al. Leptin levels reflect body lipid content in mice: evidence for diet-induced resistance to leptin action. Nat. Med 1, 1311-1314 (1995).

5. De Vos, P., Saladin, R., Auwerx, J. \& Staels, B. Induction of ob gene expression by corticosteroids is accompanied by body weight loss and reduced food intake. J. Biol. Chem. 270, 15958-15961 (1995).

6. Pickup, J. C., Chusney, G. D. \& Mattock, M. B. The innate immune response and type 2 diabetes: evidence that leptin is associated with a stress-related (acutephase) reaction. Clin. Endocrinol. 52, 107-112 (2000).

7. Clark, K. A., Shin, A. C., Sirivelu, M. P., Mohankumar, S. M. \& Mohankumar, P. S. Systemic administration of leptin decreases plasma corticosterone levels: role of hypothalamic norepinephrine. Brain Res 1195, 89-95 (2008).

8. Francis, J., MohanKumar, S. M. \& MohanKumar, P. S. Leptin inhibits norepinephrine efflux from the hypothalamus in vitro: role of gamma aminobutyric acid. Brain Res 1021, 286-291 (2004).

9. Heiman, M. L. et al. Leptin inhibition of the hypothalamic-pituitary-adrenal axis in response to stress. Endocrinology 138, 3859-3863 (1997).

10. Koch, C. E. et al. High-fat diet induces leptin resistance in leptin-deficient mice. J. Neuroendocrinol. 26, 58-67 (2014).

11. Bjorbaek, C., Elmquist, J. K., Frantz, J. D., Shoelson, S. E. \& Flier, J. S. Identification of SOCS-3 as a potential mediator of central leptin resistance. Mol. Cell 1, 619-625 (1998).

12. Banks, W. A. et al. Triglycerides induce leptin resistance at the blood-brain barrier. Diabetes 53, 1253-1260 (2004).
13. Benthem, L. et al. Excess portal venous long-chain fatty acids induce syndrome X via HPA axis and sympathetic activation. Am. J. Physiol. Endocrinol. Metab. 279, E1286-E1293 (2000).

14. Oh, I. S. et al. Molecular mechanisms associated with leptin resistance: n-3 polyunsaturated fatty acids induce alterations in the tight junction of the brain. Cell Metab. 1, 331-341 (2005).

15. Spangelo, B. L., Judd, A. M., Call, G. B., Zumwalt, J. \& Gorospe, W. C. Role of the cytokines in the hypothalamic-pituitary-adrenal and gonadal axes. Neuroimmunomodulation 2, 299-312 (1995).

16. Turnbull, A. V. \& Rivier, C. Regulation of the HPA axis by cytokines. Brain Behav. Immun. 9, 253-275 (1995)

17. Widmaier, E. P., Margenthaler, J. \& Sarel, I. Regulation of pituitary-adrenocortical activity by free fatty acids in vivo and in vitro. Prostaglandins Leukot. Essent. Fat Acids 52, 179-183 (1995).

18. Widmaier, E. P., Rosen, K. \& Abbott, B. Free fatty acids activate the hypothalamic-pituitary-adrenocortical axis in rats. Endocrinology 131, 2313-2318 (1992).

19. Clark, K. A., MohanKumar, S. M., Kasturi, B. S. \& MohanKumar, P. S. Effects of central and systemic administration of leptin on neurotransmitter concentrations in specific areas of the hypothalamus. Am. J. Physiol. Regul. Integr. Comp. Physiol. 290, R306-R312 (2006).

20. Paxinos GaW, C. The Rat Brain in Stereotaxic Coordinates, 2nd edn (Academic San Diego, CA, 1987).

21. Contreras, G. A., O'Boyle, N. J., Herdt, T. H. \& Sordillo, L. M. Lipomobilization in periparturient dairy cows influences the composition of plasma nonesterified fatty acids and leukocyte phospholipid fatty acids. J. Dairy Sci. 93, 2508-2516 (2010).

22. Ricci, M. R. \& Levin, B. E. Ontogeny of diet-induced obesity in selectively bred Sprague-Dawley rats. Am. J. Physiol. Regul. Integr. Comp. Physiol. 285, R610-R618 (2003).

23. Cunningham, E. T. Jr. \& Sawchenko, P. E. Anatomical specificity of noradrenergic inputs to the paraventricular and supraoptic nuclei of the rat hypothalamus. J. Comp. Neurol. 274, 60-76 (1988).

24. Levin, B. E. \& Dunn-Meynell, A. A. Defense of body weight depends on dietary composition and palatability in rats with diet-induced obesity. Am. J. Physiol. Regul. Integr. Comp. Physiol. 282, R46-R54 (2002).

25. Levin, B. E., Dunn-Meynell, A. A., Balkan, B. \& Keesey, R. E. Selective breeding for diet-induced obesity and resistance in Sprague-Dawley rats. Am. J. Physiol. 273 (2 Pt 2), R725-R730 (1997).

26. Levin, B. E., Richard, D., Michel, C. \& Servatius, R. Differential stress responsivity in diet-induced obese and resistant rats. Am. J. Physiol. Regul. Integr. Comp. Physiol. 279, R1357-R1364 (2000).

27. Michel, C., Dunn-Meynell, A. \& Levin, B. E. Reduced brain CRH and GR mRNA expression precedes obesity in juvenile rats bred for diet-induced obesity. Behav. Brain Res 154, 511-517 (2004).

28. Michel, C., Levin, B. E. \& Dunn-Meynell, A. A. Stress facilitates body weight gain in genetically predisposed rats on medium-fat diet. Am. J. Physiol. Regul. Integr. Comp. Physiol. 285, R791-R799 (2003).

29. Chan, J. L., Wong, S. L. \& Mantzoros, C. S. Pharmacokinetics of subcutaneous recombinant methionyl human leptin administration in healthy subjects in the fed and fasting states: regulation by gender and adiposity. Clin. Pharmacokinet. 47, 753-764 (2008).

30. Wong, S. L., DePaoli, A. M., Lee, J. H. \& Mantzoros, C. S. Leptin hormonal kinetics in the fed state: effects of adiposity, age, and gender on endogenous leptin production and clearance rates. J. Clin. Endocrinol. Metab. 89, 2672-2677 (2004).

31. Kawakami, A. et al. Leptin inhibits and ghrelin augments hypothalamic noradrenaline release after stress. Stress 11, 363-369 (2008).

32. Kutlu, S. et al. Leptin modulates noradrenaline release in the paraventricular nucleus and plasma oxytocin levels in female rats: a microdialysis study. Brain Res 1317, 87-91 (2010).

33. Sherin, J. E. \& Nemeroff, C. B. Post-traumatic stress disorder: the neurobiological impact of psychological trauma. Dialog. Clin. Neurosci. 13, 263-278 (2011).

34. Barnes, M. J., Rogers, R. C., Van Meter, M. J. \& Hermann, G. E. Co-localization of TRHR1 and LepRb receptors on neurons in the hindbrain of the rat. Brain Res 1355, 70-85 (2010).

35. Grill, H. J. et al. Evidence that the caudal brainstem is a target for the inhibitory effect of leptin on food intake. Endocrinology 143, 239-246 (2002).

36. Hay-Schmidt, A., Helboe, L. \& Larsen, P. J. Leptin receptor immunoreactivity is present in ascending serotonergic and catecholaminergic neurons of the rat. Neuroendocrinology 73, 215-226 (2001). 
37. Maniscalco, J. W. \& Rinaman, L. Systemic leptin dose-dependently increases STAT3 phosphorylation within hypothalamic and hindbrain nuclei. Am. J. Physiol. Regul. Integr. Comp. Physiol. 306, R576-R585 (2014).

38. Ferrante, A. W. Jr. Obesity-induced inflammation: a metabolic dialogue in the language of inflammation. J. Intern Med 262, 408-414 (2007).

39. Shoelson, S. E., Herrero, L. \& Naaz, A. Obesity, inflammation, and insulin resistance. Gastroenterology 132, 2169-2180 (2007).

40. Dodington, D. W., Desai, H. R. \& Woo, M. JAK/STAT-emerging players in metabolism. Trends Endocrinol. Metab. 29, 55-65 (2018).

41. Levin, B. E., Dunn-Meynell, A. A. \& Banks, W. A. Obesity-prone rats have normal blood-brain barrier transport but defective central leptin signaling before obesity onset. Am. J. Physiol. Regul. Integr. Comp. Physiol. 286, R143-R150 (2004).

42. Cheng, L. et al. Arachidonic acid impairs hypothalamic leptin signaling and hepatic energy homeostasis in mice. Mol. Cell Endocrinol. 412, 12-18 (2015).
43. Park, H. K. \& Ahima, R. S. Physiology of leptin: energy homeostasis, neuroendocrine function and metabolism. Metabolism 64, 24-34 (2015).

44. Sahu, A. Minireview: a hypothalamic role in energy balance with special emphasis on leptin. Endocrinology 145, 2613-2620 (2004).

45. Arase, K., York, D. A., Shimizu, H., Shargill, N. \& Bray, G. A. Effects of corticotropinreleasing factor on food intake and brown adipose tissue thermogenesis in rats. Am. J. Physiol. 255(3 Pt 1), E255-E259 (1988).

46. Olson, B. R., Drutarosky, M. D., Stricker, E. M. \& Verbalis, J. G. Brain oxytocin receptors mediate corticotropin-releasing hormone-induced anorexia. Am. J. Physiol. 260(2 Pt 2), R448-R452 (1991).

47. Masaki, T. et al. Corticotropin-releasing hormone-mediated pathway of leptin to regulate feeding, adiposity, and uncoupling protein expression in mice. Endocrinology 144, 3547-3554 (2003). 